\title{
A Lattice Boltzmann method with moment-based boundary conditions for rarefied flow in the slip regime
}

\author{
S. Mohammed* \\ College of Science, Department of Mathematics, \\ University of Baghdad, Baghdad, Iraq \\ T. Reis ${ }^{\dagger}$ \\ School of Computing and Mathematical Sciences, \\ University of Greenwich, SE10 9LS, UK
}

(Dated: September 14, 2021)

\begin{abstract}
A lattice Boltzmann method with moment-based boundary conditions is used to compute flow in the slip regime. Navier-Maxwell slip conditions and Burnett-order stress conditions that are consistent with the discrete velocity Boltzmann equation are imposed locally on stationary and moving boundaries. Micro Couette and micro lid driven driven cavity flows are studied numerically at Knudsen and Mach numbers of the order $\mathscr{O}\left(10^{-1}\right)$. The Couette results for velocity and the deviatoric stress at second order in Knudsen number are in excellent agreement with analytical solutions and the cavity results are in excellent agreement with existing data. The algorithm is shown to compute nonequilibrium effects in the pressure that are in very good agreement with DSMC simulations of the Boltzmann equation but not captured by the Navier-Stokes equations.
\end{abstract}

Keywords: Lattice Boltzmann, slip flow regime, moment-based boundary conditions

\footnotetext{
*seemaa.a@sc.uobaghdad.edu.iq

$\dagger$ T.Reis@Greenwich.ac.uk
} 


\section{INTRODUCTION}

The study of flows in micro-devices has received much attention in recent years [1-4]. In micro-devices the Knudsen number $K n$ (the ratio between the mean free path of particles and a characteristic macroscopic lengthscale) and the Mach number $M a$ (the ratio of the flow speed to the speed of sound) are typically of the order $\mathscr{O}\left(10^{-1}\right)$. This is known as the slip flow regime and it exhibits phenomena that is not captured by the Navier-Stokes equations. This includes narrow $(\mathscr{O}(K n)$ wide) slip boundary layers in the macroscopic velocity, known as Knudsen layers [2], and non-equilibrium effects in the pressure tensor [5]. To fully capture the flow one can solve the Boltzmann equation

$$
\frac{\partial f}{\partial t}+\mathbf{c} \cdot \nabla f=-\frac{1}{\tau}\left(f-f^{(e)}\right)
$$

where $f=f(\mathbf{x}, \mathbf{c}, t)$ is the particle velocity distribution function with velocity $\mathbf{c}$ at position $\mathbf{x}$ and time $t$. The right hand side is the BGK collision term, which assumes the relaxation of $f$ to the Maxwell-Boltzmann equilibrium $f^{(e)}$ over the collisional timescale $\tau$.

Solving equation (1) is challenging due to its high dimensionality. Simpler, lower dimensional, macroscopic equations of motion are thought to be good approximate descriptions of slip flow outside of the boundary layers as long as appropriate boundary conditions are used [6]. The three non-dimensional numbers that characterise the macroscopic flow are the Reynolds, Mach, and Knudsen numbers, which are connected by von Kármán's relation and defined to be

$$
R e=\frac{\rho u H}{\mu}, \quad M a=\frac{u}{a}, \quad K n=\sqrt{\frac{\pi \gamma}{2}} \frac{M a}{R e},
$$

where $u$ is the characteristic flow velocity, $\rho$ is the density of the fluid, $H$ the macroscopic length scale and $\mu$ is the dynamic viscosity. The speed of sound in the fluid is $a=\sqrt{\gamma R T}$ where $\gamma$ is the ratio of specific heats for an ideal monatomic gas (taken to be 5/3) and $R$ and $T$ are the gas constant and absolute temperature. Later we will use the ideal equation of state for pressure, $p=\rho R T$. The Navier-Stokes equations with Navier-Maxwell slip boundary conditions are often solved in favour of the Boltzmann equation [7]. Here, provided the velocity in the bulk flow is accurately captured, one sacrifices capturing the narrow boundary layer in favour of ease of analysis or computation.

The Navier-Stokes equations can be recovered from an asymptotic expansion of (1) to first order in $K n \ll 1$. Thus by modelling the slip flow regime using the Navier-Stokes equations one is ignoring physical contributions to non-conserved hydrodynamic moments at higher order in $K n$, as 
well as the Knudsen velocity boundary layer. These contributions can be seen and understood by taking moments in the hydrodynamic reference frame, that is with respect to the peculiar velocity. Defining the peculiar velocity $\boldsymbol{\xi}=\mathbf{c}-\mathbf{u}$, one can obtain from (1) the evolution of the pressure tensor $\mathbf{P}=\int \boldsymbol{\xi} \boldsymbol{\xi} f d \boldsymbol{\xi}[8]$

$$
\partial_{t} P_{\alpha \beta}+\partial_{\gamma}\left(u_{\gamma} \partial_{\gamma} P_{\alpha \beta}+Q_{\alpha \beta \gamma}\right)+P_{\alpha \gamma} \frac{\partial u_{\beta}}{\partial x_{\gamma}}+P_{\gamma \beta} \frac{\partial u_{\alpha}}{\partial x_{\gamma}}=-\frac{1}{\tau}\left(P_{\alpha \beta}-P_{\alpha \beta}^{(e)}\right)
$$

where $\mathbf{Q}=\int \boldsymbol{\xi} \boldsymbol{\xi} \boldsymbol{\xi} f d \boldsymbol{\xi}$. This level of detail can be appreciable for moderate Knudsen numbers (as discussed below) but can not be predicted by the Navier-Stokes model.

The importance of flows in microdevices with moving boundaries has seen the development of numerical methods for macroscopic equations in the slip flow regime and also numerical solvers for the Boltzmann equation [9-13]. Of particualr interest here, Mizzi et al. [5] computed solutions to the Navier-Stokes-Fourier equations in micro lid driven cavity flow and compared them with their DSMC results for the Boltzmann equation. Importantly, they found that while the NavierStokes-Fourier model could predict the velocity field well outside of kinetic boundary layers, it could not compute accurate solutions to other aspects of flow in the slip regime. In particular, and in agreement with Jiang et. al. [14], they showed that the non-equilibrium effects of the Knudsen number were strong near the moving wall and in the vicinity of the corners, with the NavierStokes and DSMC solvers predicting considerably different pressures. Mizzi et al. [5] argued for alternative solution methods to be developed. We show in this article that the lattice Boltzmann equation with moment-based boundary conditions can accurately capture the pressure distribution in the slip flow regime where the Navier-Stokes-Fourier equations could not.

The lattice Boltzmann method (LBM) may be considered an intermediate approach to computing slip flow. Its discrete particle velocity set restricts it to capturing only the first few moments of the Boltzmann equation (1) and, for standard integer lattices at least, it cannot capture kinetic effects in the velocity field [15]. However, the LBM will capture the bulk flow with a high degree of accuracy when supplemented with appropriate slip boundary conditions [16, 17]. Furthermore, and despite being primary used as a Navier-Stokes solver, it can compute the pressure tensor, viz the deviatoric stress, from kinetic theory with an isothermal equation of state, even with a small number of degrees of freedom [18]. The application of the LBM to the slip flow regime has received a lot of attention [16, 17, 19-25], but most studies used "kinetic-style" boundary conditions that are prone to inaccuracies in the slip at the boundaries - see Verhaeghe et. al. [17] and Reis and Dellar [16] for an overview. A two-relaxation-time collision operator can be used to remove 
or minimise such artefacts, allowing for a far more robust scheme [26, 27], yet the timescale of the relaxation of the third order moment required to minismise the error is too long to capture the deviatoric stress at appreciable Knudsen numbers [28]. Alternatively, a novel technique for implementing boundary conditions in the lattice Boltzmann method was proposed by Bennett [29] and used to impose the Navier-Maxwell conditions precisely for slip flow in microchannels by Reis and Dellar [16]. This methodology does not produce any artificial slip and can be used with any lattice Boltzmann collision operator [30-32]. It can also be used to impose boundary conditions that are consistent with the deviatoric stress and has been shown to predict non-Navier-Stokes behaviour [28, 33], but until now this has not been used to compute the slip-flow regime. Here we show that the LBM with consistent moment-based boundary conditions can accurately compute flow in the slip regime, including subtle effects in the pressure that the Navier-Stokes-Fourier systems cannot capture.

The remainder of the article is orgnaised as follows. In Section II we discuss the lattice Boltzmann framework for the slip flow regime, and we explain the boundary conditions and their implementation in Section III. Numerical results for micro Couette and mico lid driven cavity flows are shown in sections IV and V, respectively, and concluding remarks are made in Section VI.

\section{THE LATTICE BOLTZMANN METHODOLOGY}

The discrete velocity Boltzmann equation with a BGK collision operator with relaxation time $\tau$ is

$$
\frac{\partial f_{i}}{\partial t}+\mathbf{c}_{i} \cdot \nabla f_{i}=-\frac{1}{\tau}\left(f_{i}-f_{i}^{(0)}\right)
$$

where $f_{i}$ represents the particle distribution function with discrete particle velocity $\mathbf{c}_{i}$. Here, the truncated discrete velocity set $\left\{\mathbf{c}_{i} \mid i=0, \ldots, 8\right\}$ forms the integer D2Q9 lattice shown in Figure 1. The prescribed equilibria, $f_{i}^{(0)}$, are $[34,35]$

$$
f_{i}^{(0)}(\mathbf{x}, t)=\omega_{i} \rho\left(1+3 \mathbf{c}_{i} \cdot \mathbf{u}+\frac{9}{2}\left(\mathbf{c}_{i} \cdot \mathbf{u}\right)^{2}-\frac{3}{2} \mathbf{u}^{2}\right),
$$

with lattice weights

$$
\omega_{i}= \begin{cases}4 / 9, & \text { if } i=0 \\ 1 / 9, & \text { if } i=1, \ldots, 4 \\ 1 / 36, & \text { if } i=5, \ldots, 8\end{cases}
$$




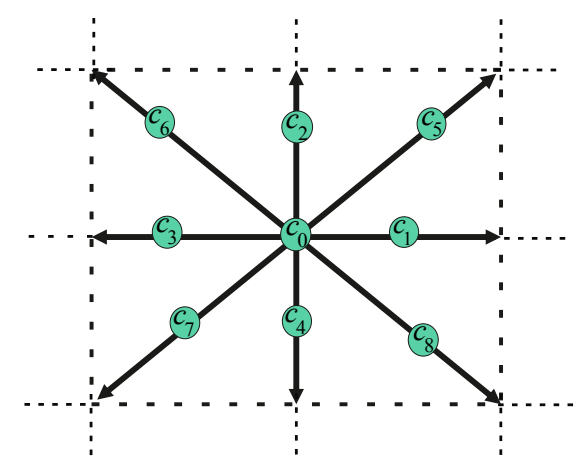

FIG. 1. The nine particle velocities and the D2Q9 lattice.

Macroscopic observables are obtained by taking discrete velocity moments of $f_{i}$. The first three moments are the hydrodynamic mass, momentum, and momentum flux, respectively:

$$
\rho=\sum_{i} f_{i} ; \quad \rho \mathbf{u}=\sum_{i} f_{i} \mathbf{c}_{i} ; \quad \boldsymbol{\Pi}=\sum_{i} f_{i} \mathbf{c}_{i} \mathbf{c}_{i}
$$

and mass and momentum are conserved by collisions:

$$
\rho=\sum_{i} f_{i}^{(0)} ; \quad \rho \mathbf{u}=\sum_{i} f_{i}^{(0)} \mathbf{c}_{i}
$$

The remaining moments are non-hydrodynamic moments (or "ghost moments") and are given by

$$
Q_{x x y}=\sum_{i} f_{i} c_{i y} c_{i x}^{2}, \quad Q_{x y y}=\sum_{i} f_{i} c_{i x} c_{i y}^{2}, \quad S_{x x y y}=\sum_{i} f_{i} c_{i x}^{2} c_{i y}^{2} .
$$

\section{A. Macroscopic equations}

Taking the first three moments of the discrete Boltzmann equation (4) yields exact conservation laws for mass and momentum, and a partial differential equation for the evolution of the flux of momentum,

$$
\begin{aligned}
\partial_{t} \rho+\nabla \cdot \rho \mathbf{u} & =0 \\
\partial_{t} \rho \mathbf{u}+\nabla \cdot \boldsymbol{\Pi} & =0 \\
\partial_{t} \boldsymbol{\Pi}+\nabla \cdot \mathbf{Q} & =-\frac{1}{\tau}\left(\boldsymbol{\Pi}-\boldsymbol{\Pi}^{(0)}\right),
\end{aligned}
$$

where $\boldsymbol{\Pi}^{(0)}=\sum_{i} f_{i}^{(0)} \mathbf{c}_{i} \mathbf{c}_{i}$ is the equilibrium momentum flux tensor and, from equation (5), is known to be

$$
\boldsymbol{\Pi}^{(0)}=\frac{\rho}{3} \mathbf{I}+\rho \mathbf{u u}
$$


We identify the pressure as $p=\rho / 3=\rho R T$, i.e an ideal equation of state. The right hand side of equation (12) includes the deviatoric stress $\Gamma=\Pi^{0}-\Pi$, which one usually approximates to first order in relaxation time $\tau$ (or Knudsen number) using a Chapman-Enskog analysis. Instead, the stress can be obtained by taking moments with respect to the discrete peculiar velocity $\boldsymbol{\xi}_{i}=\mathbf{c}_{i}-\mathbf{u}$. The pressure tensor $\mathbf{P}=\sum_{i} f_{i} \boldsymbol{\xi}_{i} \boldsymbol{\xi}_{i}$ in discrete kinetic theory evolves according to the same PDE found in classical kinetic theory, i.e equation (3), although without enough degrees of freedom to specify $\mathbf{Q}$ completely [8, 18, 36]. Following Dellar [18], the deviatoric stress with the D2Q9 lattice isothermal equation of state, $p=\rho R T=\rho / 3$, is $\Gamma=\rho / 3 \mathbf{I}-\mathbf{P}$ and is governed by

$$
\Gamma_{\alpha \beta}+\tau\left(\partial_{t} \Gamma_{\alpha \beta}+u_{\gamma} \partial_{\gamma} \Gamma_{\alpha \beta}+\Gamma_{\alpha \gamma} \partial_{\gamma} u_{\beta}+\Gamma_{\beta \gamma} \partial_{\gamma} u_{\alpha}\right)=\mu\left(\partial_{\beta} u_{\alpha}+\partial_{\alpha} u_{\beta}\right)
$$

where $\mu=\rho \tau / 3$ is the dynamic viscosity. It has been assumed that the third order moment remains

close to its equilibrium, which is $\mathscr{O}\left(M a^{3}\right)$, and thus has been neglected (and we note that the equilibrium part of $\mathbf{Q}$ is zero in continuous kinetic theory) [18]. It is noted again that this is an isothermal LBM.

Clearly, the stress embedded in the D2Q9 discrete velocity model includes contributions beyond what is captured by the Navier-Stokes equations. The Navier-Stokes relation would only be recovered if the terms multiplied by $\tau$ on the left hand side of equation (14) vanish. Instead, the stress includes nonequilibrium effects from the Boltzmann equation. While these contributions may be negligible for very small Knudsen numbers, they can be appreciable in the slip flow regime or when $\tau$ is not small $[16,28]$.

\section{B. From discrete Boltzmann to lattice Boltzmann}

To solve equation (4) numerically we integrate both sides along a characteristic for time, $\Delta t$, to obtain

$$
f_{i}\left(\mathbf{x}+\mathbf{c}_{i} \Delta t, t+\Delta t\right)-f_{i}(\mathbf{x}, t)=-\frac{1}{\tau} \int_{0}^{\Delta t}\left(f_{i}-f_{i}^{(0)}\right) d s
$$

The integral on the right side is approximated using the Trapezoidal rule to give a fully discrete and implicit system of algebraic equations:

$$
\begin{aligned}
& f_{i}\left(\mathbf{x}+\mathbf{c}_{i} \Delta t, t+\Delta t\right)-f_{i}(\mathbf{x}, t)= \\
& -\frac{\Delta t}{2 \tau}\left(f_{i}\left(\mathbf{x}+\mathbf{c}_{i} \Delta t, t+\Delta t\right)-f_{i}^{(0)}\left(\mathbf{x}+\mathbf{c}_{i} \Delta t, t+\Delta t\right)\right)-\frac{\Delta t}{2 \tau}\left(f_{i}(\mathbf{x}, t)-f_{i}^{(0)}(\mathbf{x}, t)\right)+\mathscr{O}\left(\Delta t^{3}\right) .
\end{aligned}
$$


This second order implicit system is converted into an explicit algorithm using He's et.al. [37] variable change

$$
\bar{f}_{i}(\mathbf{x}, t)=f_{i}(\mathbf{x}, t)+\frac{\Delta t}{2 \tau}\left(f_{i}(\mathbf{x}, t)-f_{i}^{(0)}(\mathbf{x}, t)\right)
$$

to obtain the second order in space and time lattice Boltzmann equation for $\bar{f}_{i}$ :

$$
\bar{f}_{i}\left(\mathbf{x}+\mathbf{c}_{i} \Delta t, t+\Delta t\right)-\bar{f}_{i}(\mathbf{x}, t)=-\frac{\Delta t}{(\tau+\Delta t / 2)}\left(\bar{f}_{i}(\mathbf{x}, t)-f_{i}^{(0)}(\mathbf{x}, t)\right)+\mathscr{O}\left(\Delta t^{3}\right) .
$$

Recall that the grid spacing and time step are connected by the lattice speed $c=(\Delta x / \Delta t) \gg 1$.

Density, momentum and momentum flux are computed from $\bar{f}_{i}$ using the variable change (17),

$$
\begin{aligned}
& \rho=\sum_{i} f_{i}=\sum_{i} \bar{f}_{i}, \quad \rho \mathbf{u}=\sum_{i} \bar{f}_{i} \mathbf{c}_{i}=\sum_{i} f_{i} \mathbf{c}_{i}, \\
& \bar{\Pi}=\sum_{i} \bar{f}_{i} \mathbf{c}_{i} \mathbf{c}_{i}=\sum_{i} f_{i}+\sum_{i} \frac{\Delta t}{2 \tau}\left(f_{i}-f_{i}^{(0)}\right)=\frac{(2 \tau+\Delta t)}{2 \tau} \Pi-\frac{\Delta t}{2 \tau} \boldsymbol{\Pi}^{(0)} .
\end{aligned}
$$

Thus, the deviatoric stress is given in terms of barred quantities as

$$
\boldsymbol{\Gamma}=\boldsymbol{\Pi}^{0}-\boldsymbol{\Pi}=\frac{2 \tau\left(\boldsymbol{\Pi}^{(0)}-\overline{\boldsymbol{\Pi}}\right)}{(2 \tau+\Delta t)} .
$$

\section{HYDRODYNAMIC BOUNDARY CONDITIONS FOR THE SLIP FLOW REGIME}

Modelling microflows at small Knudsen numbers with macroscopic conservation laws can capture phenomena outside of the velocity boundary layers provided suitable boundary conditions are used. We consider flat walls that may move tangentially only. In the slip flow regime, the tangential velocity at a wall can be modelled with the Navier-Maxwell condition, which says the amount of slippage is proportional to the shear at the wall [6],

$$
u_{x}=U_{m}-\left.\sigma K n H \frac{\partial u_{x}}{\partial y}\right|_{w a l l} .
$$

Here, we have taken the liberty to let the subscripts $x$ and $y$ denote the tangential and normal directions at a flat wall, respectively. In equation (22) $U_{m}$ is the velocity of the wall and $\sigma$ is the (streamwise momentum) accommodation coefficient. In what follows we use $\sigma=1$. The coefficient $K n H$, where $H$ is the characteristic macroscopic lengthscale, defines the mean free path. The kinematic condition states that $u_{y}=0$.

In Section II A we saw that the stress embedded in the lattice Boltzmann model is given by equation (14). At a boundary we assume there is no dependence on time nor tangential coordinate, 
$\partial_{t}=\partial_{x}=0$. Noting that the normal velocity is zero by the kinematic condition, the components of stress at a boundary are

$$
\begin{aligned}
\Gamma_{x x}+2 \tau \Gamma_{x y} \frac{\partial u_{x}}{\partial y} & =0, \\
\Gamma_{y y} & =0, \\
\Gamma_{x y}-\mu \frac{\partial u_{x}}{\partial y} & =0 .
\end{aligned}
$$

Equations (24)-(25) represent the shear stress and the normal component of the stress at a boundary respectively, and they are the same as in the Navier-Stokes equations. Equation (23) shows the tangential component of the stress at a boundary. This would be zero in the Navier-Stokes equation but at $O\left(\tau^{2}\right)$ it is proportional to the square of the shear. Thus special consideration of the tangential stress at the boundary is needed in lattice Boltzmann computations.

\section{A. Moment-based boundary conditions for the lattice Boltzmann equation}

The moment-based approach for implementing boundary conditions for the lattice Boltzmann equation is a general methodology that imposes constraints directly and precisely upon moments of the LBE and then translates them into conditions for the unknown distribution functions at a boundary [29]. The unknowns are the incoming $f_{i}$ (or $\bar{f}_{i}$ ), i.e the $f_{i}$ with velocity $\mathbf{c}_{i}$ pointing into the fluid domain, and clearly one should have as many boundary moment conditions as there are unknown $f_{i}$, and these moments need to be linearly independent at a boundary. For the D2Q9 lattice with boundaries aligned with grid points three conditions are needed for three unknowns.

We proceed by illustrating the method using a horizontal boundary at the north of the domain.

Here, the functions $\bar{f}_{4}, \bar{f}_{7}$, and $\bar{f}_{8}$ are unknown and need to be supplied to the algorithm (see Figure 1). These unknowns appear in the moments in one of three linear combinations, as shown in Table I. Thus one must impose a condition on one moment from each row of Table I and then solve for the unknown $f_{i}$ (noting the definitions given in equations (7) and (9)). For the second order discretisation (18), the conditions need to be expressed in terms of "barred" quantities using the transformation (17). Considering we are computing solutions to hydrodynamic flow equations we choose to impose hydrodynamic constraints. For the slip flow regime considered here we have conditions for the two components of momentum,

$$
\rho u_{y}=0, \quad \rho u_{x}=\rho U_{m}-\left.\rho \sigma K n H \frac{\partial u_{x}}{\partial y}\right|_{\text {wall }},
$$




\begin{tabular}{c|c}
\hline Moments & Combination of unknowns at north boundary \\
\hline$\rho, \rho u_{y}, \Pi_{y y}$ & $f_{4}+f_{7}+f_{8}$ \\
\hline$\rho u_{x}, \Pi_{x y}, Q_{x y y}$ & $f_{7}-f_{8}$ \\
\hline$\Pi_{x x}, Q_{x x y}, S_{x x y y}$ & $f_{7}+f_{8}$ \\
\hline
\end{tabular}

TABLE I. Moment combinations for unknown $f_{i}$ at the north boundary.

and also the tangential component of the momentum flux (and hence stress) - the only hydrodynamic moment in the final row of Table I.

Equation (23) for the tangential stress can be rewritten using equation (25) as

$$
\Gamma_{x x}=-\frac{2 \tau}{\mu} \Gamma_{x y}^{2}
$$

and since $\Gamma_{x x}=\Pi_{x x}^{(0)}-\Pi_{x x}$, we have the boundary condition for the momentum flux moment of the LBE:

$$
\Pi_{x x}=\frac{\rho}{3}+\rho u_{x}^{2}+\frac{2 \tau}{\mu} \Gamma_{x y}^{2}
$$

This condition, together with the two in equation (26), give our three linear independent moment constraints at the boundary. Using the transformation (17), these are easily converted into conditions on "barred" moments:

$$
\begin{aligned}
& \rho \bar{u}_{y}=0, \\
& \rho \bar{u}_{x}=\rho U_{m}+\frac{2 \tau \rho \sigma K n H}{\mu(2 \tau+1)} \bar{\Pi}_{x y}, \\
& \bar{\Pi}_{x x}=\frac{\rho}{3}+\rho u_{x}^{2}+\frac{4 \tau^{2}}{\mu(2 \tau+1)} \bar{\Pi}_{x y}^{2},
\end{aligned}
$$

where we have used equations (21) and (25) to write the shear derivative in terms of locallyavailable moments (noting that $\Pi_{x y}^{(0)}=\rho u_{x} u_{y}=0$ at the boundary). The moments $\rho$ and $\bar{\Pi}_{x y}$ in the boundary conditions above can be written in terms of imposed conditions and known (incoming) distribution functions:

$$
\begin{aligned}
\rho & =\rho u_{y}+\bar{f}_{0}+\bar{f}_{1}+\bar{f}_{3}+2\left(\bar{f}_{2}+\bar{f}_{5}+\bar{f}_{6}\right) ; \\
& =\bar{f}_{0}+\bar{f}_{1}+\bar{f}_{3}+2\left(\bar{f}_{2}+\bar{f}_{5}+\bar{f}_{6}\right) ; \\
\bar{\Pi}_{x y} & =-\rho u_{x}+\bar{f}_{1}-\bar{f}_{3}+2 \bar{f}_{5}-2 \bar{f}_{6} .
\end{aligned}
$$


Simply solving the linear system (29) for the three unknowns yields

$$
\begin{aligned}
& \bar{f}_{4}=-\bar{\Pi}_{x x}+\bar{f}_{1}+\bar{f}_{3}+\bar{f}_{2}+2\left(\bar{f}_{5}+\bar{f}_{6}\right), \\
& \bar{f}_{7}=\frac{1}{2}\left(\bar{\Pi}_{x x}-\rho u_{x}\right)-\bar{f}_{3}-\bar{f}_{6}, \\
& \bar{f}_{8}=\frac{1}{2}\left(\rho u_{x}+\bar{\Pi}_{x x}\right)-\bar{f}_{1}-\bar{f}_{5} .
\end{aligned}
$$

We note that most previous applications of moment-based boundary conditions [16, 29, 30, 3840] impose a Navier-Stokes stress condition, $\Gamma_{x x}=0$ at a boundary but this has been shown to be inconsistent with the underlying moment system $[28,33]$.

\section{MICRO-COUETTE FLOW}

We use the lattice Boltzmann equation to compute solutions to planar micro-Couette flow with the Navier-Maxwell slip condition (22) and Burnett stress condition (23) imposed on the top and bottom boundaries using the moment-based approach described in Section III A. The top wall moves horizontally with velocity $U_{m}$ while the parallel bottom wall, which is a distance $H$ away, is stationary. The domain is periodic in the flow direction. Four different values of the Knudsen number are used: $K n=0.01,0.05,0.1$ and 0.2 ; and our results are compared with the analytical solution of the Navier-Stokes equations with the aforementioned boundary conditions, assuming the flow is steady $\left(\partial_{t}=0\right)$, incompressible $(\rho=$ constant $)$, and unidirectional $(u=u(y))$ :

$$
u(y)=\frac{U_{m}}{(1+2 K n)}\left(\left(\frac{y}{H}\right)+K n\right) .
$$

In the plots that follow the velocity has been scaled with the wall moving velocity, $U_{m}$, and the normal coordinate with the channel height, $H$.

Figure 2 plots the numerical and analytical solution for the velocity when $K n=0.05$. For clarity of visualisation, a $1 \times 17$ grid is used in the plot but errors smaller than $\mathscr{O}\left(10^{-10}\right)$ were observed on just 3 grid points. Figure 3 plots the velocity profiles at different $K n$ and shows the increasing slip at the boundaries as we increase the Knudsen number. In all cases the LBM results are grid independent, agree well with the benchmark data [22, 23, 25] and, moreover, with the analytical solution.

Figure 4 plots the computed and analytical solution of the tangential component of the deviatoric stress down the center of the channel, $\Gamma_{x x}(0.5, y)$, when $K n=0.05$ and $M a=0.09$. The 


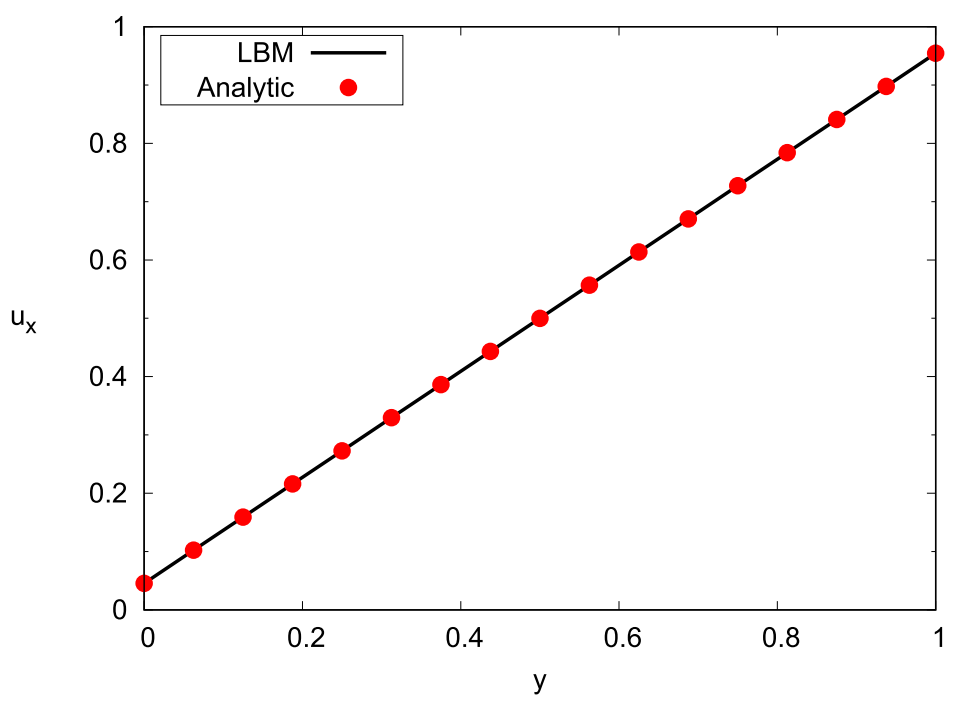

FIG. 2. Numerical and analytical solutions for the velocity in micro-Couette flow with $K n=0.05$ and $M a=0.09$.

analytical solution is

$$
\Gamma_{x x}=-2 \tau \mu\left(\frac{U_{m}}{H(1+2 K n)}\right)^{2},
$$

where we have used equation (14) noting that the flow is steady $\left(\partial_{t}=0\right)$, unidirectional $(u=u(y))$, and the flow velocity is given by equation (31). The computed solution with the Navier-Stokes stress condition $\Gamma_{x x}=0$ that has often been used with the moment-based approach [16, 30, 38, $39,41]$ is also shown. The resolution is the same as above and the results are grid independent. The figure illustrates the excellent agreement between the numerical and analytical solutions for $\Gamma_{x x}$ using the Navier-Maxwell slip (22) and stress moment-based boundary conditions (23). We remark that when the Navier-Stokes condition is used the stress is constrained to be zero at the boundaries, and this is in contradiction the the underling PDE moment system, and hence the spurious numerical boundary layers observed in Figure 4.

\section{MICRO LID-DRIVEN CAVITY FLOW}

The methodology is applied to two dimensional micro-lid driven cavity flow, with the top wall of a square box of side $L$ moving horizontally with constant velocity $U_{m}$ while the other boundaries are stationary, see Figure 5. The Navier-Maxwell slip condition (22) and the Burnett stress condition (23) are applied to all boundaries. A grid of size $65 \times 65$ was used in the simulations 


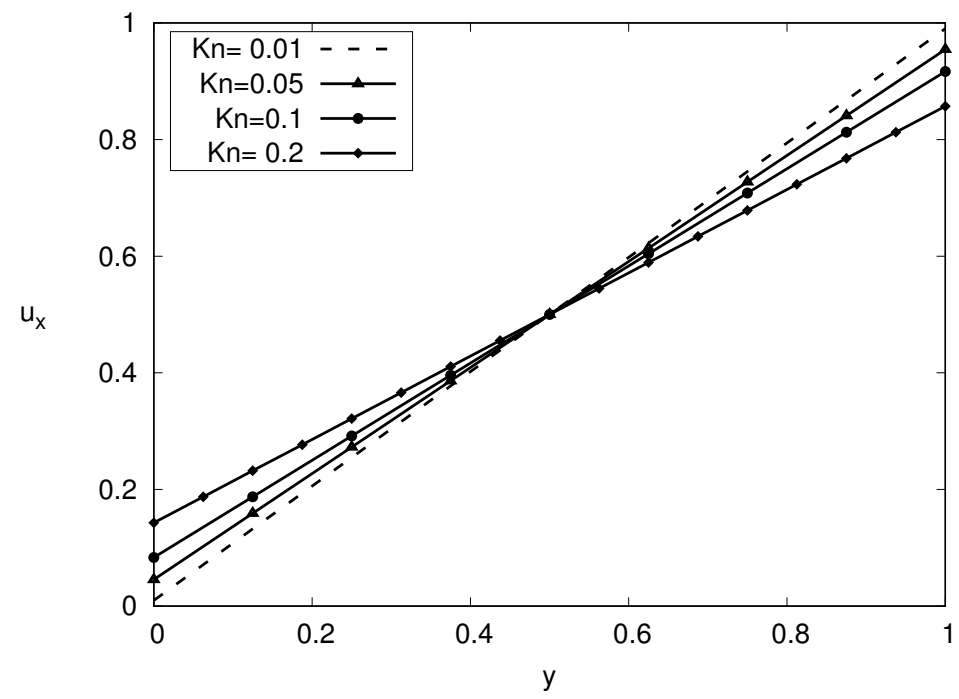

FIG. 3. Velocity profiles of mico-Couette flow at $K n=0.01,0.05,0.1,0.2$ with $M a=0.09$.

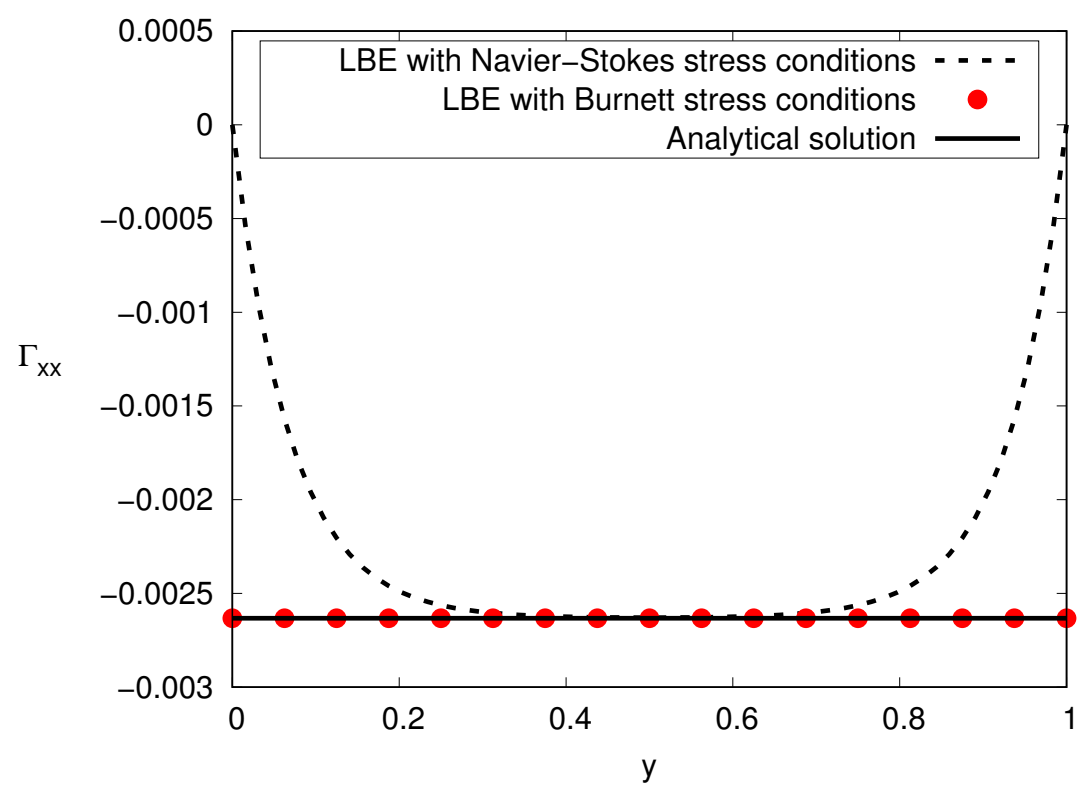

FIG. 4. Numerical and analytical solutions for the deviatoric stress $\Gamma_{x x}(0.5, y)$ in micro-Couette flow with $K n=0.05$ and $M a=0.09$. Numerical results for the consistent Burnett-order (23) and inconsistent NavierStokes $\left(\Gamma_{x x}=0\right)$ order stress boundary conditions are shown.

shown here with no discernible differences in the velocity field on finer grids and second order convergence has been observed from $17 \times 17$ grids. In the plots that follow the velocity has been scaled with the moving wall velocity, $U_{m}$, and the coordinates with the channel height, $L$.

Figures 6 and 7 show the effect of the Knudsen number on the behaviour of the velocity when the Mach number is fixed at $M a=0.09$. The wall slippage increases with $K n$ and the respective 


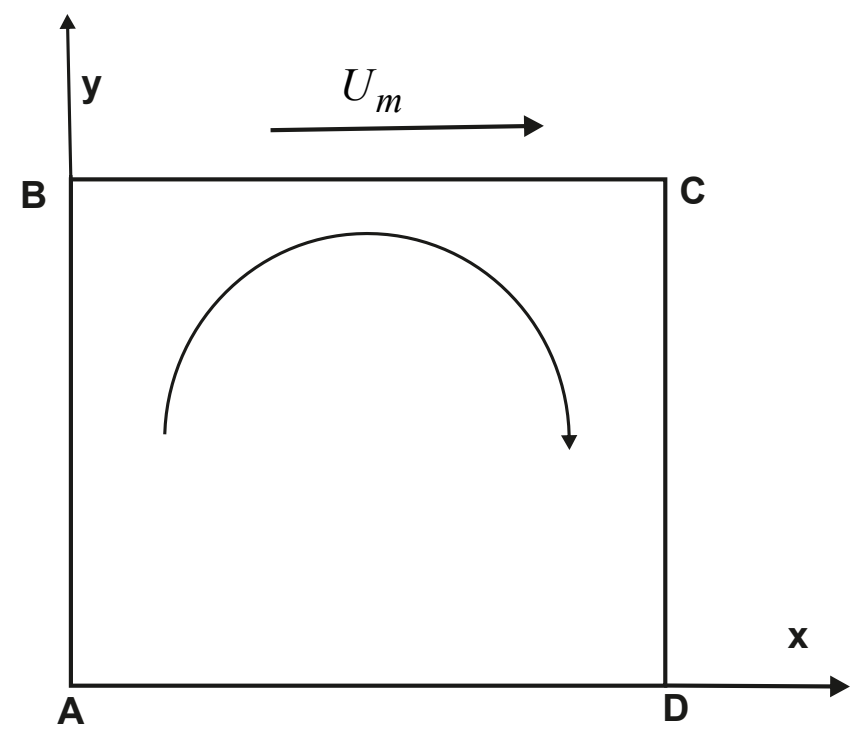

FIG. 5. Schematic diagram of lid-driven cavity flow

turning points are smaller in magnitude, in agreement with existing results $[9,11,22,25]$.

We plot in Figure 8 the streamlines when $K n=0.1$ and 0.001 ; the latter being used to highlight vanishingly small slip lengths. The secondary vortices in the corners that appear for the case of no-slip are not detected when there is significant wall slip (increasing Knudsen number), and the location of the primary vortex travel towards the centre of the cavity as we increase $K n$, in agreement with the results of Ogata and Kawaguchi [9]. For more quantitative measures, Table II show the maximum value of the primary stream function, $\Psi_{\max }$, and its location. Our data shows that $\Psi_{\max }$ decreases when the Knudsen number increases with fixed $M a$ (meaning that $R e$ is decreasing by von-Kármán's relation, equation (2)), and the location of the maximum stream function creeps downwards with higher $K n$ and thus more slip.

Finally, the normalised pressure $P=p / p_{0}$ along the surface of the cavity when $K n=0.05$ is shown in Figure 9, and when $K n=0.1$ in Figure 10. In these figures the coordinate $S$ refers to the distance along the perimeter of the cavity in the clockwise direction from the origin $(0,0)$, denoted by $A$ in Figure 5. A $129 \times 129$ grid was used and no discernible difference was observed on finer grids. The influence of the Knudsen number and rarefaction on the pressure distribution is seen and in agreement with Jian et al [14]. Moreover, our results are in very good quantitative agreement with the hard sphere DSMC results of Mizzi et.al. [5]. The computed solutions of the Navier-Stokes-Fourier equations with slip conditions of Mizzi et.al. [5] are also shown in Figures 9 and 10. It is noteworthy that the solutions based on the Navier-Stokes-Fourier model, which has 


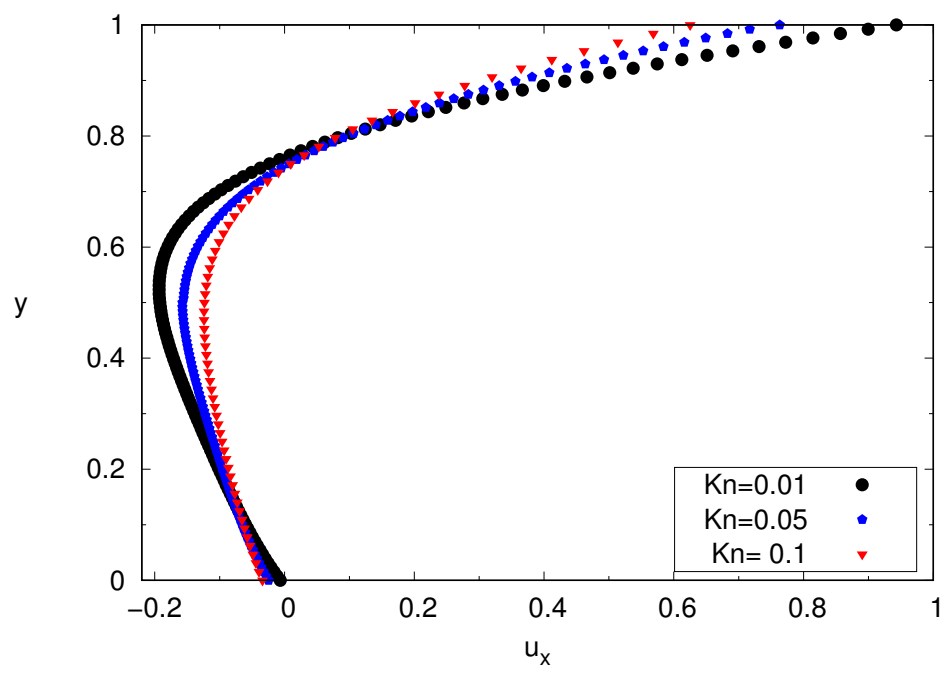

FIG. 6. Horizontal velocity profiles $u_{x}(x, 0.5)$ at different Knudsen numbers when $M a=0.09$.

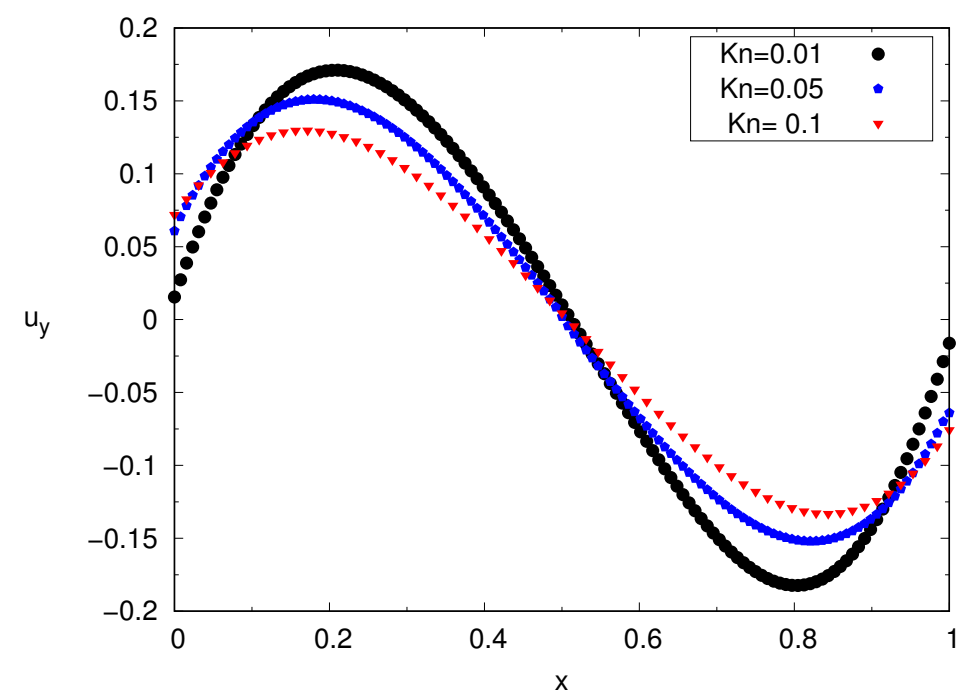

FIG. 7. Vertical velocity profiles $u_{y}(0.5, y)$ at different Knudsen numbers when $M a=0.09$.

the normal pressure tensor $-p \mathbf{I}$, over predicts the pressure greatly, whereas the lattice Boltzmann model with the prescribed boundary conditions has a pressure evolution equation that agrees with kinetic theory and computes solutions in agreement with the DSMC results.

\section{CONCLUSION}

The lattice Boltzmann method has been used to compute near-continuum flow. Navier-Maxwell slip velocity conditions and Burnett-order deviatoric stress conditions were imposed on moving and stationary boundaries to capture Knudsen phenomena using a moment-based approach. Al- 

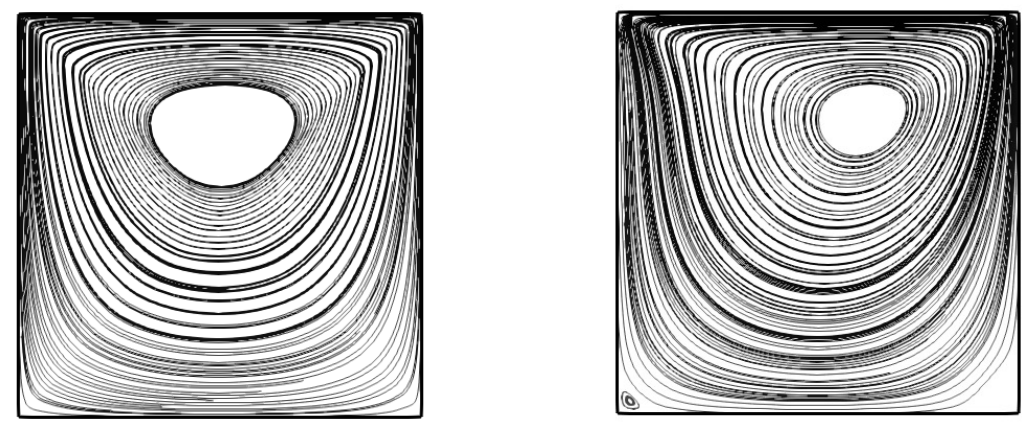

FIG. 8. Streamlines in micro lid driven cavity flow when $M a=0.09$ and $K n=0.1$ (left) and $K n=0.001$.

\begin{tabular}{|c|c|c|}
\hline$K n$ & $\Psi_{\max }$ & $(x, y)$ \\
\hline 0.001 & 0.1047 & $(0.60,0.73)$ \\
0.01 & 0.0951 & $(0.50,0.77)$ \\
0.05 & 0.0789 & $(0.50,0.74)$ \\
0.10 & 0.0681 & $(0.50,0.71)$ \\
0.20 & 0.0635 & $(0.49,0.63)$ \\
\hline
\end{tabular}

TABLE II. The maximum value of the streamfunction and its location for various $K n$ when $M a=0.09$.

though the lattice Boltzmann method does not compute Knudsen boundary layers, it can predict the slip flow regime outside of these boundary layers very well. This has previously been shown for pressure driven micro channel flows using the moment-based approach by Reis and Dellar [16] and extended here to more complicated flows and boundaries. The influence of the Knudsen number on the behaviour of the micro lid driven cavity has been examined, with the cross channel velocity profiles flattening and the primary vortex creeping towards the cavity centre as $K n$ increases.

Furthermore, the D2Q9 lattice Boltzmann can compute subtle kinetic phenomena in its nonconserved hydrodynamic moments, even though it does not capture Knudsen boundary layers. The pressure tensor embedded in the moments of the LBM approximates that of the BGK Boltzmann 


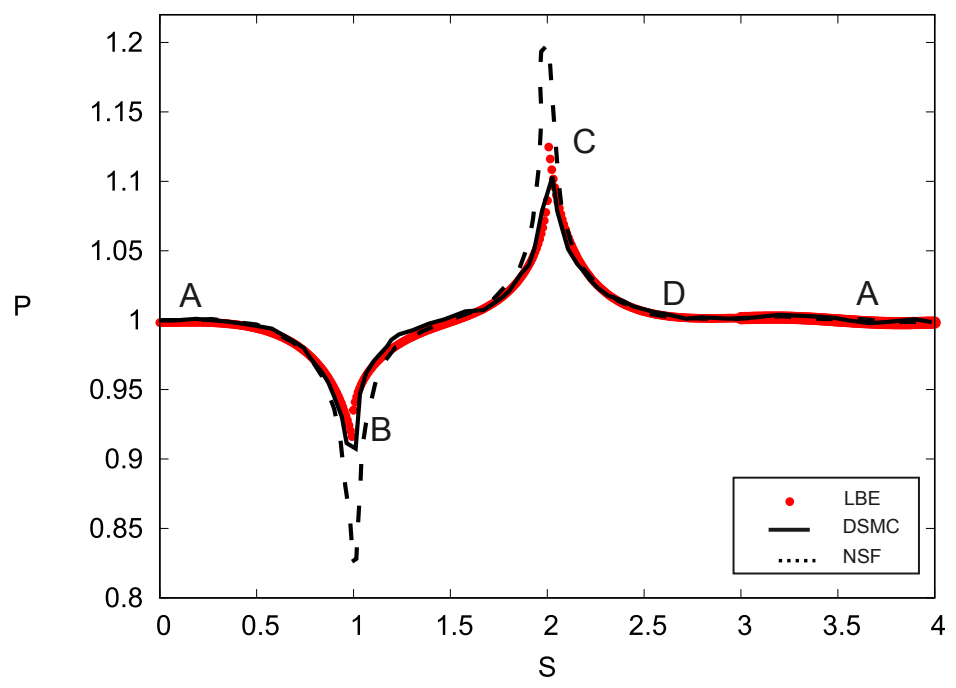

FIG. 9. Non dimensional pressure distribution $\mathrm{P}=p / p_{0}$ along the cavity walls when $K n=0.05$ and $M a=$ 0.09. Mizzi's et al. [5] DSMC (line) and Navier-Stokes-Fourier (dashed) results are also shown

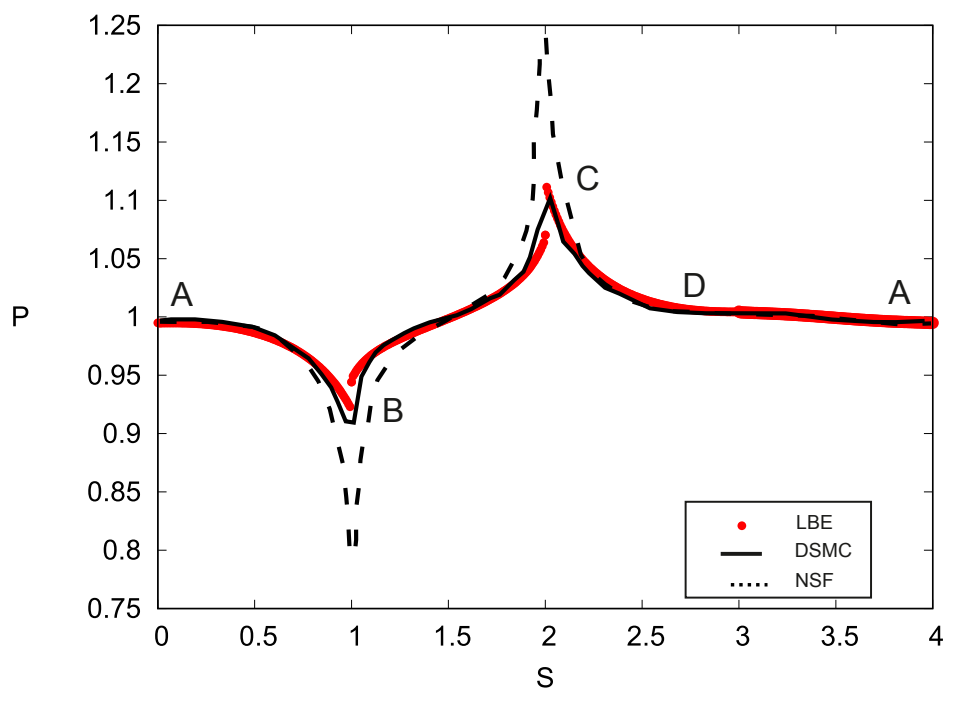

FIG. 10. Non dimensional pressure distribution $\mathrm{P}=p / p_{0}$ along the cavity walls when $K n=0.1$ and $M a=$ 0.09. Mizzi's et al. [5] DSMC (line) and Navier-Stokes-Fourier (dashed) results are also shown

equation when the Mach number is small but fixed and the flow is isothermal with the equation of state $p=\rho R T[18,28,33]$. Spurious numerical boundary layers in computations of the stress can appear in simple flows if constraints inconsistent with the pressure tensor are imposed but these can be removed using the moment-based method. We remark that all results presented here converged under mesh refinement with fixed non-dimensional numbers. 
We have shown that the LBM can predict near-wall nonequilibrium phenomena in the pressure. Where simulations of the Navier-Stokes-Fourier equations over predict the pressure considerably, the LBM computations are in very good agreement with the DSMC results of Mizzi et. al [5]. While the model considered here does not include temperature, extending the methodology to thermal flows is a prospect for future work, and following Krastins et. al. [32], the extension to other lattices in three-dimensions is relatively straightforward.

\section{ACKNOWLEDGMENTS}

This research forms part of the actives of the UK Consortium on Mesoscopic Engineering Science (UKCOMES) [grant number EP/L00030X/1].

[1] A. T. Conlisk, Essentials of micro-and nanofluidics: with applications to the biological and chemical sciences (Cambridge University Press, 2012).

[2] G. Karniadakis, A. Beskok, and N. Aluru, Microflows and nanoflows: fundamentals and simulation, Vol. 29 (Springer Science \& Business Media, 2006).

[3] W. M. Zhang, G. Meng, and X. Wei, Microfluidics and nanofluidics 13, 845 (2012).

[4] H. Xia, J. Wu, J. Zheng, J. Zhang, and Z. Wang, Lab. Chip 21, 1241 (2021).

[5] S. Mizzi, D. R. Emerson, S. K. Stefanov, R. W. Barber, and J. M. Reese, Journal of computational and theoretical nanoscience 4, 817 (2007).

[6] N. Hadjiconstantinou, Phys. Fluids 18, 111301 (2006).

[7] E. B. Arkilic, M. A. Schmidt, and K. S. Breuer, J. Micro syst. 6, 167 (1997).

[8] E. Ikenberry and C. Truesdell, J. Rational Mech. Anal 5, 1 (1956).

[9] Y. Ogata and T. Kawaguchi, Journal of Fluid Science and Technology 6, 215 (2011).

[10] M. Darbandi, H. Mahboubi Fouladi, M. Zakyani, and G. E. Schneider, in Proceedings of the International Conference on Mechanical Engineering and MechatronicsToronto (2013).

[11] S. Mukherjee, V. Shahabi, R. Gowtham, K. Rajan, and R. Velamati, Journal of Applied Fluid Mechanics 12, 1575 (2019).

[12] S. Naris, D. Valougeorgis, D. Kalempa, and F. Sharipov, Phys. Fluids 17, 100607 (2005).

[13] J. Lopez-L. and R. Velasco, Physica A: Stat. Mech. and its Applications 274, 454 (1999). 
[14] J. Z. Jiang, J. Fan, and C. Shen, in AIP Conference Proceedings, Vol. 663 (American Institute of Physics, 2003) pp. 784-791.

[15] X. He, Q. Zou, L. S. Luo, and M. Dembo, J. Stat. Phys. 87, 115 (1997).

[16] T. Reis and P. J. Dellar, Phys Fluids 24, 112001 (2012).

[17] F. Verhaeghe, L. S. Luo, and B. Blanpain, J. Comput. Phys. 228, 147 (2009).

[18] P. J. Dellar, SIAM Sci. Comput. 36, A2507 (2014).

[19] X. Nie, G. D. Doolen, and S. Chen, J. Stat. Phys. 107, 279 (2002).

[20] C. Lim, C. Shu, X. Niu, and Y. Chew, Phys. Fluids 14, 2299 (2002).

[21] S. Succi, Phys. Rev. lett. 89, 064502 (2002).

[22] G. Tang, W. Tao, and Y. He, Phys. Fluids 17, 058101 (2005).

[23] Z. Guo, C. Zheng, and B. Shi, Phys. Rev. E 77, 036707 (2008).

[24] L. Szalmás, Phys. Rev. E 73, 066710 (2006).

[25] A. Perumal, G. V. Kumar, and A. K. Dass, CFD lett 2, 75 (2010).

[26] G. Silva and V. Semião, Phys. Rev. E 96, 013311 (2017).

[27] G. Silva, Phys. Rev. E 98, 023302 (2018).

[28] T. Reis, SIAM J. on Sci., Comput. 42, B397 (2020).

[29] S. Bennett, A lattice Boltzmann model for diffusion of binary gas mixtures, Ph.D. thesis, University of Cambridge (2010).

[30] S. Mohammed, D. Graham, and T. Reis, Phys. Fluids 32, 025104 (2020).

[31] S. Mohammed, D. Graham, and T. Reis, Comput. Fluids. (2018).

[32] I. Krastins, A. Kao, K. Pericleous, and T. Reis, Int. J. Numer. Meth. Fluids 92, 1948 (2020).

[33] T. Reis, Commun. Comput. Phys 27, 167 (2020).

[34] X. He and L. S. Luo, Phys. Rev. E 56, 6811 (1997).

[35] Y. Qian, D. d'Humières, and P. Lallemand, Europhys. Lett. 17, 479 (1992).

[36] S. Chapman and T. G. Cowling, The mathematical theory of non-uniform gases: an account of the kinetic theory of viscosity, thermal conduction and diffusion in gases ((Cambridge Univ Press, Cambridge, England, 1970).

[37] X. He, X. Shan, and G. Doolen, Phys. Rev. E 57, R13 (1998).

[38] R. Allen and T. Reis, Prog. Comp. Fluid Dyn.: An Int. J. 16, 216 (2016).

[39] S. Mohammed and T. Reis, Arch. Mech. Eng. 64, 57 (2017).

[40] A. Hantsch, T. Reis, and U. Gross, J. Comput. Multiphase Flows 7, 1 (2015). 
[41] S. Bennett, P. Asinari, and P. J. Dellar, Int. J. Numer. Meth. Fluids 69, 171 (2012). 